\title{
Environmental Impacts Predictions via Kinetic Modelling
}

\author{
JULIEN DECLERCQ, MICHAEL HERRELL AND ROBERT \\ BOWELL
}

SRK Consulting

Presenting Author: jdeclercq@srk.co.uk

Numerical predictions of long term mine water quality are commonly based on weathering/solute release rates derived from kinetic laboratory testwork such as humidity cell tests or field tests, such as barrel tests. These weathering rates are then scaled up to provide estimates of future field conditions. These scaled values, a generally grossly overpredicting field concentrations and need to be controlled via the use of thermodynamic calculations. Allowing the, via the use of PHREEQC or other calculator, the reduction of the elemental concentration via the precipitation of selected secundary phases.

While thermodynamic equilibrium chemistry, the basis for these models, has strong theoretical foundations, this approach has equally strong shortcomings. For instance, most silicate do not reach equilibrium under normal Earth surface conditions. As such, kinetic modelling, e.g. the use of kinetic rate equations controlling the dissolution and precipitation mechanisms of the multimineral system forming the waste storage facilities present on most mine site would more accurately reflect a real chemistry.

In this study we have applied rate equations from litterature data to predict the leachate obtained in Humidity Cell Tests and Barrel Tests of known mineralogical composition. This acted as a calibration and validation of our methodology, before applying it to the predictions of waste storage facilities, waste rock dumps and tailings facilities. These results were compared to the more classic thermodynamic equilibrium calculations.

An example of $\mathrm{CO}_{2}$ storage prediction in mine waste was also carried out to elucidate the timescale involved in the uptake of $\mathrm{CO}_{2}$ in mine waste weathering and under which conditions this could be consistantly applied in the industry. 